\title{
An analytic formula for Macdonald polynomials
}

Une formule analytique pour les polynômes de Macdonald

\author{
Michel Lassalle and Michael Schlosser
}

5 mai 2003

\begin{abstract}
We give the explicit analytic development of any Jack or Macdonald polynomial in terms of elementary (resp. modified complete) symmetric functions. These two developments are obtained by inverting the Pieri formula.
\end{abstract}

\section{Résumé}

Nous donnons le développement analytique explicite de tout polynôme de Jack ou de Macdonald sur les fonctions symétriques élémentaires (resp. complètes modifiées). Nous obtenons ces deux développements par inversion de la formule de Pieri.

\section{Version française abrégée}

Au milieu des années cinquante, Hua introduisait les polynômes zonaux et posait le problème d'en obtenir un développement analytique explicite [1]. En dépit de nombreuses recherches, cette question est demeurée ouverte.

Elle est désormais formulée dans le cadre plus général des polynômes de Macdonald. Les polynômes zonaux sont en effet un cas particulier des polynômes de Jack, qui sont eux-mêmes un cas limite des polynômes de Macdonald.

Ces polynômes sont indexés par les partitions d'entiers. Ils forment une base de l'algèbre des fonctions symétriques à coefficients rationnels en deux paramètres $q$, $t$, et généralisent notamment les fonctions de Schur, les polynômes de Hall-Littlewood, et les polynômes de Jack.

Les polynômes de Macdonald ont été déterminés par Lapointe, Lascoux et Morse [5], qui les ont exprimés comme déterminants. Cependant les entrées de ces déterminants sont des quantités combinatoires qui ne peuvent être en général exprimées analytiquement.

On ne disposait donc jusqu'ici d'aucune formule analytique explicite pour les polynômes de Jack et de Macdonald. On ignorait notamment leur développement sur les bases classiques, sauf dans deux cas particuliers : lorsque la partition indexante est de longueur deux [2, ou lorsqu'elle est de longueur trois [6], ainsi que dans les situations duales où les parts sont au plus égales à 2 ou 3. 
Le but de cette Note est de présenter une solution générale à ce problème. Pour tout polynôme de Macdonald nous obtenons deux développements analytiques explicites. Le premier se fait sur les fonctions symétriques élémentaires. Le second sur les fonctions symétriques "complètes modifiées", dont le développement sur toute base classique est connu [7.

\section{Introduction}

In the fifties, Hua posed the problem of finding an explicit analytic formula for the "zonal polynomials" he had just introduced [1]. In spite of many efforts this question has remained open.

Hua's problem is now better understood in the more general framework of Macdonald polynomials. Zonal polynomials are indeed a special case of Jack polynomials, which in turn are obtained from Macdonald polynomials by taking a particular limit.

Macdonald polynomials are indexed by partitions. They form a basis of the algebra of symmetric functions with rational coefficients in two parameters $q, t$, and generalize Schur functions, Hall-Littlewood polynomials and Jack polynomials.

Lapointe, Lascoux and Morse [5] gave a determinantal expression for Macdonald polynomials. However their method does not lead to an explicit analytic formula, since the entries of these determinants are combinatorial quantities which in general cannot be analytically explicited.

Thus Hua's problem kept open for Macdonald polynomials. Their analytic expansion was only known when the length of the indexing partition is two [2], or three [6], and in the dual cases corresponding to partitions with parts at most equal to 2 or 3 .

The aim of this Note is to present a general solution to this problem. We give two explicit analytic developments for any Macdonald polynomial. One of them is made in terms of elementary symmetric functions. The other one is made in terms of "modified complete" symmetric functions, which have themselves a known development in terms of any classical basis [7].

Our method relies on the inversion of the "Pieri matrix". This is an infinite transition matrix which has been analytically explicited by Macdonald [8]. We compute its inverse by the operator method of Krattenthaler [3, 4, such as adapted to the multivariate case by the second author 9 .

Detailed proofs will be given in a forthcoming paper.

\section{Macdonald Polynomials}

The standard reference for Macdonald polynomials is Chapter 6 of [8]

Let $X=\left\{x_{1}, x_{2}, x_{3}, \ldots\right\}$ be an infinite set of indeterminates (an alphabet) and $\mathcal{S}$ the corresponding algebra of symmetric functions with coefficients in Q. Elementary symmetric functions $e_{k}(X)$, complete symmetric functions $h_{k}(X)$ and power sums $p_{k}(X)$ form three algebraic bases of $\mathcal{S}$. 
Let $q, t$ be two indeterminates. We define $(a ; q)_{0}:=1,(a ; q)_{k}:=\prod_{i=0}^{k-1}\left(1-a q^{i}\right)$, for $k \geq 1$, and $(a ; q)_{\infty}:=\prod_{i \geq 0}\left(1-a q^{i}\right)$.

A partition $\lambda=\left(\lambda_{1}, \ldots, \lambda_{n}\right)$ is a finite weakly decreasing sequence of positive integers, called parts. The number $n=l(\lambda)$ of parts is called the length of $\lambda$. For any integer $i \geq 1, m_{i}(\lambda):=\operatorname{card}\left\{j: \lambda_{j}=i\right\}$ is the multiplicity of $i$ in $\lambda$. Clearly $l(\lambda)=\sum_{i \geq 1} m_{i}(\lambda)$. We shall also write $\lambda=\left(1^{m_{1}}, 2^{m_{2}}, 3^{m_{3}}, \ldots\right)$.

Let $\mathrm{Q}[q, t]$ be the algebra of rational functions in $q, t$, and $\mathrm{Sym}=\mathcal{S} \otimes \mathrm{Q}[q, t]$ the algebra of symmetric functions with coefficients in $\mathrm{Q}[q, t]$. This algebra is endowed with some scalar product for which Macdonald polynomials $P_{\lambda}(X ; q, t)$ form an orthogonal basis. Let $Q_{\lambda}(X ; q, t)=b_{\lambda}(q, t) P_{\lambda}(X ; q, t)$ be the dual basis, with $b_{\lambda}(q, t)$ specified in [8], p. 339, Equ. (6.19).

For any $k \geq 0$ the "modified complete" symmetric function $g_{k}(X ; q, t)$ is defined by the generating series

$$
\prod_{i \geq 1} \frac{\left(t u x_{i} ; q\right)_{\infty}}{\left(u x_{i} ; q\right)_{\infty}}=\sum_{k \geq 0} u^{k} g_{k}(X ; q, t)
$$

Then it is known ([8], p. 329, Equ. (5.5)) that the Macdonald polynomial associated with a row partition $(k)$ is given by $Q_{(k)}(q, t)=g_{k}(q, t)$.

The symmetric functions $g_{k}(q, t)$ form an algebraic basis of Sym. They may be expanded in terms of any classical basis. This development is explicitly given in [8] (p. 311 and 314) in terms of power sums and monomial symmetric functions, and in [7] (Sec. 10, p. 237) for other classical bases.

The parameters $q, t$ being kept fixed, we shall write $P_{\mu}$ for $P_{\mu}(q, t)$ (resp. $Q_{\mu}$ for $\left.Q_{\mu}(q, t)\right)$.

\section{Pieri formula}

Let $u_{1}, \ldots, u_{n}$ be $n$ indeterminates and $\mathrm{N}$ the set of nonnegative integers. For $\theta=$ $\left(\theta_{1}, \ldots, \theta_{n}\right) \in \mathbf{N}^{n}$ let $|\theta|=\sum_{i=1}^{n} \theta_{i}$ and define

$$
d_{\theta_{1}, \ldots, \theta_{n}}\left(u_{1}, \ldots, u_{n}\right):=\prod_{k=1}^{n} \frac{(t ; q)_{\theta_{k}}}{(q ; q)_{\theta_{k}}} \frac{\left(q^{|\theta|+1} u_{k} ; q\right)_{\theta_{k}}}{\left(q^{|\theta|} t u_{k} ; q\right)_{\theta_{k}}} \prod_{1 \leq i<j \leq n} \frac{\left(t u_{i} / u_{j} ; q\right)_{\theta_{i}}}{\left(q u_{i} / u_{j} ; q\right)_{\theta_{i}}} \frac{\left(q^{-\theta_{j}+1} u_{i} / t u_{j} ; q\right)_{\theta_{i}}}{\left(q^{-\theta_{j}} u_{i} / u_{j} ; q\right)_{\theta_{i}}} .
$$

Macdonald polynomials satisfy a Pieri formula ([8, p. 340, Equ. (6.24) (ii)), which may be analytically explicited as follows ([8], p. 342, Example 2(b)).

Theorem 3.1 (Macdonald). Let $\lambda=\left(\lambda_{1}, \ldots, \lambda_{n}\right)$ be an arbitrary partition with length $n$ and $\lambda_{n+1} \in \mathrm{N}$. For all $1 \leq k \leq n$ define $u_{k}:=q^{\lambda_{k}-\lambda_{n+1}} t^{n-k}$. We have

$$
Q_{\left(\lambda_{1}, \ldots, \lambda_{n}\right)} Q_{\left(\lambda_{n+1}\right)}=\sum_{\theta \in \mathrm{N}^{n}} d_{\theta_{1}, \ldots, \theta_{n}}\left(u_{1}, \ldots, u_{n}\right) Q_{\left(\lambda_{1}+\theta_{1}, \ldots, \lambda_{n}+\theta_{n}, \lambda_{n+1}-|\theta|\right)} .
$$

This Pieri formula defines an infinite transition matrix. Indeed let $\operatorname{Sym}(n+1)$ denote the algebra of symmetric polynomials in $n+1$ independent variables with coefficients in $\mathrm{Q}[q, t]$. Then the Macdonald polynomials $\left\{Q_{\lambda}, l(\lambda) \leq n+1\right\}$ form a basis of $\operatorname{Sym}(n+1)$, and so do the products $\left\{Q_{\mu} Q_{(k)}, l(\mu) \leq n, k \geq 0\right\}$. 


\section{Main results}

Let $u=\left(u_{1}, \ldots, u_{n}\right)$ and $v=\left(v_{1}, \ldots, v_{n}\right)$ be $2 n$ indeterminates. We denote by $\Delta(v)$ the Vandermonde determinant $\prod_{1 \leq i<j \leq n}\left(v_{i}-v_{j}\right)$. For $a, b$ two indeterminates and $\theta=$ $\left(\theta_{1}, \ldots, \theta_{n}\right) \in \mathbf{N}^{n}$ we define

$$
\begin{aligned}
c_{\theta}^{(a, b)}(u ; v):=\prod_{k=1}^{n} b^{\theta_{k}} \frac{(a / b ; a)_{\theta_{k}}}{(a ; a)_{\theta_{k}}} \frac{\left(a u_{k} ; a\right)_{\theta_{k}}}{\left(a b u_{k} ; a\right)_{\theta_{k}}} \prod_{1 \leq i<j \leq n} \frac{\left(a u_{i} / b u_{j} ; a\right)_{\theta_{i}}}{\left(a u_{i} / u_{j} ; a\right)_{\theta_{i}}} \frac{\left(a^{-\theta_{j}} b u_{i} / u_{j} ; a\right)_{\theta_{i}}}{\left(a^{-\theta_{j}} u_{i} / u_{j} ; a\right)_{\theta_{i}}} \\
\times \frac{1}{\Delta(v)} \operatorname{det}\left[v_{i}^{n-j}\left(1-b^{j-1} \frac{1-b v_{i}}{1-v_{i}} \prod_{k=1}^{n} \frac{u_{k}-v_{i}}{b u_{k}-v_{i}}\right)\right]_{1 \leq i, j \leq n} .
\end{aligned}
$$

We have the following result.

Theorem 4.1. Let $\lambda=\left(\lambda_{1}, \ldots, \lambda_{n+1}\right)$ be an arbitrary partition with length $n+1$. For all $1 \leq k \leq n$ define $u_{k}:=q^{\lambda_{k}-\lambda_{n+1}} t^{n-k}$. We have

$$
Q_{\left(\lambda_{1}, \ldots, \lambda_{n+1}\right)}=\sum_{\theta \in \mathbb{N}^{n}} c_{\theta_{1}, \ldots, \theta_{n}}^{(q, t)}\left(u_{1}, \ldots, u_{n} ; u_{1} q^{\theta_{1}}, \ldots, u_{n} q^{\theta_{n}}\right) Q_{\left(\lambda_{n+1}-|\theta|\right)} Q_{\left(\lambda_{1}+\theta_{1}, \ldots, \lambda_{n}+\theta_{n}\right)} .
$$

The reader will easily check that for $n=1$ we obtain the result previously given in [2]. For $n=2$ we recover the formula announced in a previous note by the first author [6].

Sketch of proof of Theorem 4.1. We invert the infinite transition matrix defined by the Pieri formula in Theorem [3.1. We accomplish this by applying Krattenthaler's [3, 4] operator method for proving matrix inversions as adapted to the multivariate case by the second author 9 .

Let $\mathbf{Z}$ denote the set of integers and let $\beta=\left(\beta_{1}, \ldots, \beta_{n}\right), \kappa=\left(\kappa_{1}, \ldots, \kappa_{n}\right), \gamma=$ $\left(\gamma_{1}, \ldots, \gamma_{n}\right) \in Z^{n}$. Defining

$$
f_{\beta \kappa}:=c_{\beta_{1}-\kappa_{1}, \ldots, \beta_{n}-\kappa_{n}}^{(q, t)}\left(u_{1} q^{\kappa_{1}+|\kappa|}, \ldots, u_{n} q^{\kappa_{n}+|\kappa|} ; u_{1} q^{\beta_{1}+|\kappa|}, \ldots, u_{n} q^{\beta_{n}+|\kappa|}\right),
$$

and

$$
g_{\kappa \gamma}:=d_{\kappa_{1}-\gamma_{1}, \ldots, \kappa_{n}-\gamma_{n}}\left(u_{1} q^{\gamma_{1}+|\gamma|}, \ldots, u_{n} q^{\gamma_{n}+|\gamma|}\right),
$$

our method yields that the infinite lower-triangular multidimensional matrices $\left(f_{\beta \kappa}\right)_{\beta, \kappa \in Z^{n}}$ and $\left(g_{\kappa \gamma}\right)_{\kappa, \gamma \in Z^{n}}$ are inverses of each other, i. e., the orthogonality relation

$$
\sum_{\kappa \in Z^{n}} f_{\beta \kappa} g_{\kappa \gamma}=\delta_{\beta \gamma}
$$

for all $\beta, \gamma \in \mathbf{Z}^{n}$, holds.

It is immediately clear from (44) that if $\left(w_{\kappa}\right)_{\kappa \in Z^{n}}$ and $\left(y_{\kappa}\right)_{\kappa \in Z^{n}}$ are two multivariate sequences of indeterminates, then

$$
\sum_{\beta \in Z^{n}} f_{\beta \kappa} w_{\beta}=y_{\kappa} \quad \text { for all } \kappa \in Z^{n},
$$


if and only if

$$
\sum_{\kappa \in Z^{n}} g_{\kappa \gamma} y_{\kappa}=w_{\gamma} \quad \text { for all } \gamma \in Z^{n}
$$

subject to convergence.

Now if in Theorem 3.1, we replace $u_{i}$ by $u_{i} q^{\gamma_{i}+|\gamma|}, \lambda_{i}$ by $\lambda_{i}+\gamma_{i}$, for $i=1, \ldots, n$, and $\lambda_{n+1}$ by $\lambda_{n+1}-|\gamma|$, then we see (after shifting the summation indices) that (6) holds for $y_{\kappa}=Q_{\left(\lambda_{1}+\kappa_{1}, \ldots, \lambda_{n}+\kappa_{n}, \lambda_{n+1}-|\kappa|\right)}$ and $w_{\gamma}=Q_{\left(\lambda_{1}+\gamma_{1}, \ldots, \lambda_{n}+\gamma_{n}\right)} Q_{\left(\lambda_{n+1}-|\gamma|\right)}$, with $g_{\kappa \gamma}$ given as in (3). Thus, with $f_{\beta \kappa}$ given as in (2), we immediately establish (15) for above values of $y_{\kappa}$ and $w_{\gamma}$. Setting now $\kappa_{i}=0$ for $i=1, \ldots, n$ gives (11).

It is known $\left([8]\right.$, p. 327) that there exists an automorphism $\omega_{q, t}$ of Sym such that

$$
\omega_{q, t}\left(Q_{\lambda}(q, t)\right)=P_{\lambda^{\prime}}(t, q), \quad \omega_{q, t}\left(g_{n}(q, t)\right)=e_{n},
$$

with $\lambda^{\prime}$ the partition conjugate to $\lambda$, whose parts are given by $m_{k}\left(\lambda^{\prime}\right)=\lambda_{k}-\lambda_{k+1}$. Applying this automorphism to the previous theorem we obtain the following equivalent result.

Theorem 4.2. Let $\lambda=\left(1^{m_{1}}, 2^{m_{2}}, \ldots,(n+1)^{m_{n+1}}\right)$ be an arbitrary partition consisting of parts at most equal to $n+1$. For all $1 \leq k \leq n$ define $u_{k}:=q^{n-k} t^{\sum_{j=k}^{n} m_{j}}$. We have

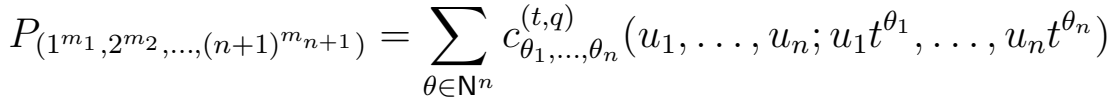

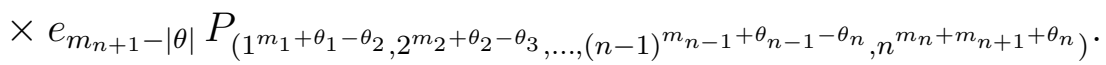

\section{$5 \quad$ Analytic developments}

Theorem 4.1 (resp. Theorem 4.2) immediately generates the analytic development of any Macdonald polynomial in terms of the symmetric functions $g_{k}$ (resp. the elementary symmetric functions $e_{k}$ ), which form an algebraic basis of Sym.

Indeed for any partition $\mu=\left(\mu_{1}, \ldots, \mu_{l}\right)$ with length $l>1$ let us write

$$
c_{\theta_{1}, \ldots, \theta_{l-1}}(\mu):=c_{\theta_{1}, \ldots, \theta_{l-1}}^{(q, t)}\left(u_{1}, \ldots, u_{l-1} ; u_{1} q^{\theta_{1}}, \ldots, u_{l-1} q^{\theta_{l-1}}\right)
$$

with $u_{k}:=q^{\mu_{k}-\mu_{l}} t^{l-k-1}$. Let $\mathrm{M}^{(n)}$ be the set of lower triangular $n \times n$ matrices with nonnegative integers. By an obvious iteration we deduce the following analytic expansion of any Macdonald polynomial.

Theorem 5.1. Let $\lambda=\left(\lambda_{1}, \ldots, \lambda_{n+1}\right)$ be an arbitrary partition with length $n+1$. For any $\theta=(\theta(i, j))_{i, j=1}^{n} \in \mathrm{M}^{(n)}$, let us consider a sequence of $n$ partitions $\{\mu(\theta, k), 1 \leq k \leq n\}$ where $\mu(\theta, k)$ has length $k+1$ and is defined by

$$
\mu(\theta, k)_{i}=\lambda_{i}+\sum_{j=k+1}^{n} \theta(j, i) \quad(1 \leq i \leq k+1) .
$$


We have

$$
Q_{\lambda}=\sum_{\theta \in \mathrm{M}^{(n)}} \prod_{k=1}^{n} c_{\theta(k, 1) \ldots \theta(k, k)}(\mu(\theta, k)) \prod_{k=0}^{n} g_{\lambda_{k+1}+\sum_{j=k+1}^{n} \theta(j, k+1)-\sum_{j=1}^{k} \theta(k, j)} .
$$

For any partition $\mu=\left(1^{m_{1}}, 2^{m_{2}}, \ldots, l^{m_{l}}\right)$ having its parts at most equal to $l$, let us write

$$
C_{\theta_{1}, \ldots, \theta_{l-1}}(\mu):=c_{\theta_{1}, \ldots, \theta_{l-1}}^{(t, q)}\left(u_{1}, \ldots, u_{l-1} ; u_{1} t^{\theta_{1}}, \ldots, u_{l-1} t^{\theta_{l-1}}\right)
$$

with $u_{k}:=q^{l-k-1} t^{\sum_{j=k}^{l-1} m_{j}}$. By duality we deduce the following analytic expansion of Macdonald polynomials in terms of elementary symmetric functions.

Theorem 5.2. Let $\lambda=\left(1^{m_{1}}, 2^{m_{2}}, \ldots,(n+1)^{m_{n+1}}\right)$ be an arbitrary partition consisting of parts at most equal to $n+1$. For any $\theta \in \mathrm{M}^{(n)}$ let us consider a sequence of $n$ partitions $\{\mu(\theta, k), 1 \leq k \leq n\}$ where $\mu(\theta, k)$ has parts at most equal to $k+1$ and is defined by

$$
\begin{aligned}
m_{i}(\mu(\theta, k)) & =m_{i}+\sum_{j=k+1}^{n}(\theta(j, i)-\theta(j, i+1)) \quad(1 \leq i \leq k) \\
m_{k+1}(\mu(\theta, k)) & =\sum_{j=k+1}^{n+1} m_{j}+\sum_{j=k+1}^{n} \theta(j, k+1) .
\end{aligned}
$$

We have

$$
P_{\lambda}=\sum_{\theta \in \mathrm{M}^{(n)}} \prod_{k=1}^{n} C_{\theta(k, 1) \ldots \theta(k, k)}(\mu(\theta, k)) \prod_{k=0}^{n} e_{\sum_{j=k+1}^{n+1} m_{j}+\sum_{j=k+1}^{n} \theta(j, k+1)-\sum_{j=1}^{k} \theta(k, j)} .
$$

Remark : The expression given by Theorem 5.1 can be written in terms of raising operators ([8], p. 9). Writing $g_{\mu}=\prod_{k \geq 1} g_{\mu_{k}}$, the raising operators $R_{i j}$ act as follows : $R_{i j} g_{\mu}=g_{\mu_{1}} \ldots g_{\mu_{i}+1} \ldots g_{\mu_{j}-1} \ldots$ Then $\prod_{k=0}^{n} g_{\lambda_{k+1}+\sum_{j=k+1}^{n} \theta(j, k+1)-\sum_{j=1}^{k} \theta(k, j)}$ is exactly $\left(\prod_{1 \leq i<j \leq n+1} R_{i j}^{\theta(j-1, i)}\right) g_{\lambda}$.

\section{Jack polynomials}

Jack polynomials are the limit of Macdonald polynomials when $t \rightarrow 1$, with $q=t^{\alpha}$ and $\alpha$ some positive real number ([8], p. 376). We note $P_{\lambda}=\lim _{t \rightarrow 1} P_{\lambda}\left(t^{\alpha}, t\right)$ and $Q_{\lambda}=$ $\lim _{t \rightarrow 1} Q_{\lambda}\left(t^{\alpha}, t\right)$.

Let $(u)_{k}$ be the classical raising factorial defined by $(u)_{k}=\prod_{i=1}^{k}(u+i-1)$. Let $u=\left(u_{1}, \ldots, u_{n}\right)$ and $v=\left(v_{1}, \ldots, v_{n}\right)$ be $2 n$ indeterminates. For $\theta=\left(\theta_{1}, \ldots, \theta_{n}\right) \in \mathbf{N}^{n}$ and any indeterminate $a$ define

$$
\begin{aligned}
& c_{\theta}^{(a)}(u ; v):=\prod_{k=1}^{n} \frac{(1-a)_{\theta_{k}}}{\theta_{k} !} \frac{\left(u_{k}+1\right)_{\theta_{k}}}{\left(u_{k}+1+a\right)_{\theta_{k}}} \prod_{1 \leq i<j \leq n} \frac{\left(u_{i}-u_{j}+1-a\right)_{\theta_{i}}}{\left(u_{i}-u_{j}+1\right)_{\theta_{i}}} \frac{\left(u_{i}-u_{j}-\theta_{j}+a\right)_{\theta_{i}}}{\left(u_{i}-u_{j}-\theta_{j}\right)_{\theta_{i}}} \\
& \times \frac{1}{\Delta(v)} \operatorname{det}\left[v_{i}^{n-j}-\left(v_{i}-a\right)^{n-j} \frac{v_{i}+a}{v_{i}} \prod_{k=1}^{n} \frac{v_{i}-u_{k}}{v_{i}-u_{k}-a}\right]_{1 \leq i, j \leq n} .
\end{aligned}
$$


Our theorems in Section 4 give rise, after letting $q=t^{\alpha}$ and taking the limit $t \rightarrow 1$, to the following results.

Theorem 6.1. Let $\lambda=\left(\lambda_{1}, \ldots, \lambda_{n+1}\right)$ be an arbitrary partition with length $n+1$. For all $1 \leq k \leq n$ define $u_{k}:=\lambda_{k}-\lambda_{n+1}+(n-k) / \alpha$. We have

$$
Q_{\left(\lambda_{1}, \ldots, \lambda_{n+1}\right)}=\sum_{\theta \in \mathbb{N}^{n}} c_{\theta_{1}, \ldots, \theta_{n}}^{(1 / \alpha)}\left(u_{1}, \ldots, u_{n} ; u_{1}+\theta_{1}, \ldots, u_{n}+\theta_{n}\right) Q_{\left(\lambda_{n+1}-|\theta|\right)} Q_{\left(\lambda_{1}+\theta_{1}, \ldots, \lambda_{n}+\theta_{n}\right)} .
$$

Theorem 6.2. Let $\lambda=\left(1^{m_{1}}, 2^{m_{2}}, \ldots,(n+1)^{m_{n+1}}\right)$ be an arbitrary partition consisting of parts at most equal to $n+1$. For all $1 \leq k \leq n$ define $u_{k}:=\sum_{j=k}^{n} m_{j}+(n-k) \alpha$. We have

$$
\begin{aligned}
& P_{\left(1^{m_{1}, 2^{m}}, \ldots,(n+1)^{m_{n+1}}\right)}=\sum_{\theta \in \mathrm{N}^{n}} c_{\theta_{1}, \ldots, \theta_{n}}^{(\alpha)}\left(u_{1}, \ldots, u_{n} ; u_{1}+\theta_{1}, \ldots, u_{n}+\theta_{n}\right)
\end{aligned}
$$

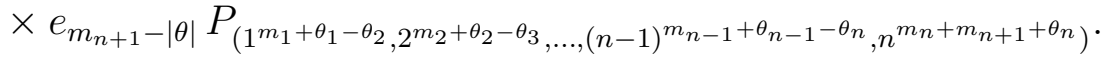

As in Section 5 these formulas generate explicit analytic developments for Jack polynomials.

Acknowledgements : The first author is pleased to thank Alain Lascoux for friendly advices.

\section{References}

[1] L.-K. Hua, Harmonic analysis of functions of several complex variables in the classical domains, American Mathematical Society, Providence, 1963.

[2] N. H. Jing, T. Józefiak, A formula for two-row Macdonald functions, Duke Math. J., 67 (1992), 377-385.

[3] C. Krattenthaler, Operator methods and Lagrange inversion, a unified approach to Lagrange formulas, Trans. Amer. Math. Soc., 305 (1988), 431-465.

[4] C. Krattenthaler, A new matrix inverse, Proc. Amer. Math. Soc., 124 (1996), 47-59.

[5] L. Lapointe, A. Lascoux, J. Morse, Determinantal expressions for Macdonald polynomials, Int. Math. Res. Not., 18 (1998), 957-978.

[6] M. Lassalle, Explicitation des polynômes de Jack et de Macdonald en longueur trois, C. R. Acad. Sci. Paris Sér. I Math., 333 (2001), 505-508.

[7] M. Lassalle, Une q - spécialisation pour les fonctions symétriques monomiales, Adv. Math., 162 (2001), 217-242.

[8] I. G. Macdonald, Symmetric functions and Hall polynomials, Clarendon Press, second edition, Oxford, 1995. 
[9] M. Schlosser, Multidimensional matrix inversions and $A_{r}$ and $D_{r}$ basic hypergeometric series, Ramanujan J., 1 (1997), 243-274.

Michel Lassalle

Centre National de la Recherche Scientifique

Institut Gaspard Monge

77454 Marne-la-Vallée Cedex, France

e-mail: lassalle @ univ-mlv.fr

http://www-igm.univ-mlv.fr/ lassalle/index.html
Michael Schlosser

Institut für Mathematik

Universität Wien

A-1090 Wien, Autriche

e-mail: schlosse @ ap.univie.ac.at

http://www.mat.univie.ac.at/ ^ schlosse/ 